\title{
Visible Light Photocatalytic Bacterial Inactivation on Titanium Dioxide Coatings ${ }^{\dagger}$
}

\author{
Vignesh Nandakumar ${ }^{1}$, Zhao Han ${ }^{1}$, Zachary Fritz ${ }^{2}$, \\ Vijay Krishna ${ }^{4}$, Ben Koopman ${ }^{3}$ and Brij Moudgil ${ }^{1 *}$ \\ ${ }^{1}$ Department of Materials Science and Engineering' University of Florida, USA \\ ${ }^{2}$ Department of Chemical Engineering, University of Florida, USA \\ ${ }^{3}$ Department of Environmental Engineering, University of Florida, USA \\ ${ }^{4}$ Department of Biomedical Engineering, Cleveland Clinic, USA
}

\begin{abstract}
The increasing number of touch surface mediated infections has steered research to look for alternative strategies that can prevent infection transmission via pathogen inactivation on surfaces. Anatase, a crystalline form of titanium dioxide, shows strong UV induced photocatalytic properties. However, nanoparticles of anatase have been found to inactivate organic contaminants in the visible spectrum. Degradation of mordant orange and inactivation of $S$. aureus was evaluated on anatase surfaces under visible light band pass filters across the visible light region. Inactivation shows a strong co-relation to the absorption spectrum of the dye/microbe on the surface of the anatase coating. The phenomenon is similar to dye sensitized solar cells and was not found to exist in coatings with a higher bandgap such as amorphous silica. Photocatalytic nano-crystalline anatase coatings hold potential as a visible light active disinfectant to inactivate microbes on touch surfaces over long periods of time.
\end{abstract}

Keywords: $\mathrm{TiO}_{2}$, visible light photocatalysis, antibacterial coatings

\section{Introduction}

Decontamination of organic and biological pollutants has gained significant attention owing to the increase in the number of infectious diseases. The increase in the number of hospital acquired infections in the last decade has led to significant research in developing alternate strategies for microbial inactivation and prevention of the transfer of such pathogens.

Hospital acquired infections or nosocomial infections are defined as infections that emanate from a stay in medical facilities and are caused by strains of microbes that have gained resistance to several bactericidal agents (Lobdell et al., 2012). These infections are extremely difficult to treat and have dramatically increased direct costs ranging from $\$ 28$ billion to $\$ 45$ billion in the United States of America (Scott II, 2009). The number of deaths caused by Methicillin Resistant Staphylococcus aureus (MRSA) account to 99,000 annually in the Unites States

\footnotetext{
Received 22 July 2015; Accepted 12 May 2016

J-STAGE Advance Publication online 9 July 2016

${ }^{1,2,3}$ Gainesville, Florida 32611, USA

4 Cleveland, Ohio 44195, USA

* Corresponding author: Brij Moudgil;

E-mail: bmoudgil@perc.ufl.edu

TEL: +1-253-846-1194 FAX: +1-352-846-1196
}

alone (Klein et al., 2007). The transfer of such pathogens causing nosocomial infections is primarily touch surface mediated (Otter et al., 2013; Weber et al., 2013). Thus there is a clear need for inactivation of microbes on these surfaces to reduce the number of nosocomial infections.

Photocatalytic coatings have shown tremendous potential for inactivating organic contaminants and bacteria (Erkan et al., 2006; Lilja et al., 2012; Wei et al., 2014). The principle of photocatalysis for microbial inactivation has been discussed in many reviews and primarily involves the generation of reactive oxygen species (ROS) including hydroxyl free radicals and superoxide free radicals (Fujishima et al., 2008; Henderson, 2011; Linsebigler et al., 1995). Titanium dioxide is one such heterogeneous photocatalyst that is an extremely efficient generator of ROS in the presence of UV light (Ishibashi et al., 2000; Konaka et al., 1999). Inactivation of various bacteria, fungi and oxidative stresses on mammalian cells has been shown due to the ROS generated on UV photo-excited $\mathrm{TiO}_{2}$ surfaces (Chen et al., 2009; Sunada et al., 2003; Tucci et al., 2013). In addition, complete degradation of organics on $\mathrm{TiO}_{2}$ surfaces ensures that there is no residue on the surface thereby reducing 'masking effects' which often reduce the efficacy of active coatings. Although $\mathrm{TiO}_{2}$ surfaces show very high potential for photocatalysis, they have only been used for surfaces that can receive a 
signtificant amount of UV light. Anatase, which is a crystallye form of $\mathrm{TiO}_{2}$, has very good photocatalytic properties but absorbs only in the near UV and UV region and has been largely ignored for use in indoor applications. This has led to extensive research into the area of visible light activated photocatalysis on modified titanium dioxide surfaces (Mitoraj et al., 2007; Yu et al., 2003). Doping of $\mathrm{TiO}_{2}$ with nitrogen and carbon has been shown to reduce the bandgap and thus enhance visible light photocatalysis. Asahi and co-workers have modified titanium dioxide with nitrogen doping to show significant increase in the photocatalytic activity under visible light (Asahi et al., 2001). Shen and group have also shown visible light photocatalysis on anatase surfaces when doped with carbon (Shen et al., 2006). The evaluation of the antibacterial performance of unmodified titanium dioxide in the presence of visible light however has largely been ignored primarily owing to a very small contribution of visible light in anatase induced photocatalysis.

However $\mathrm{TiO}_{2}$-based dye sensitized solar cell applications were based on the premise that light-absorbing organic molecules can insert electrons into the $\mathrm{TiO}_{2}$ conduction band, generate free electrons on $\mathrm{TiO}_{2}$ and activate photocatalysis (Bach et al., 1998; Krasovec et al., 2009; Oregan and Gratzel, 1991; Yu et al., 2003) We extended this concept using contaminant dyes and microbes to sensitize an otherwise inactive anatase photocatalyst to become active in the visible spectrum(Krishna et al., 2015). This property of anatase can be exploited for coating indoor surfaces which over a long period of time can lead to significant inactivation of microbes and thus reduction in the number of touch surface mediated infections.

\section{Experimental section}

\subsection{Chemical reagents}

Most Chemicals were purchased from Fisher Scientific. Mordant Orange was acquired from Sigma-Aldrich Inc. (St. Louis, MO) and anatase and silica were obtained from MKnano (M K Impex Corp. Mississauga, TO).

\subsection{Microbial culture and enumeration}

Staphylococcus aureus (ATCC 25923) was cultured in Tryptic Soy Agar (Becton, Dickinson and Company, Sparks, MD) and grown for 48 hours in the incubator at $37^{\circ} \mathrm{C}$. An individual colony from the streak plate was recovered and inoculated in Tryptic Soy broth (TSB) and grown to an $\mathrm{OD}_{600}$ of 0.6 at $37^{\circ} \mathrm{C}$ in a shaker incubator at 150 RPM. The culture was washed thrice and harvested by centrifugation at $4800 \mathrm{RCF}$ and $4{ }^{\circ} \mathrm{C}$. The final concentration for use on the tiles was set to $2 \times 10^{6}$ cells per ml. Enumeration of bacteria were performed using the pour plate method. $0.1 \mathrm{ml}$ of bacterial suspension was transferred to a petri dish and covered with melted TSA and mixed well. After the agar solidifies at room temperature the plates were incubated at $37^{\circ} \mathrm{C}$ for 24 hours.

\subsection{Coating preparation}

Coatings were made of anatase ( $7 \mathrm{~nm}$ crystallite size) and silica ( $15 \mathrm{~nm}$ crystallize size). The coatings were prepared in accordance with a protocol suggested by Bai et al (Bai et al., 2012). $10 \mathrm{mg}$ of anatase was added to $10 \mathrm{ml}$ of water to have a final concentration of $0.1 \mathrm{wt} \%$ and sonicated (Misonix Sonicator 3000, Farmingdale, NY) for 30 minutes at $90 \mathrm{~W}$ with $\mathrm{NaOH}$ as the electrostatic stabilizer at a $\mathrm{pH}$ of around 9.5. The dispersions were then applied as uniform coatings on ceramic tiles of dimensions $2.5 \mathrm{~cm} \times 2.5 \mathrm{~cm}$, with a total volume of $0.4 \mathrm{ml}$ of coating spread over the surface of the tile. A second coat of anatase was applied after the tiles were dried overnight to ensure complete coverage on the surface of the tile. Coatings of silica were prepared the same way and all coatings were stored in a dark cabinet until use.

\subsection{Estimation of bacterial inactivation}

Tiles coated with anatase and silica were inoculated with $0.1 \mathrm{ml}$ of bacteria at a starting concentration of $1-2 \times 10^{6}$ cells per $\mathrm{ml}$. The tiles were then exposed to a light intensity of $1.8 \mathrm{~W} / \mathrm{m}^{2}$ under fluorescent lamps (General Electric model T8 Ultramax F28T8-SPX41) for $18 \mathrm{~h}$ at $23{ }^{\circ} \mathrm{C}$ and $40 \%$ RH. A dark control with anatase double coat was also included for the experiments. Microbial recovery from tiles was performed by the sonication of the inoculated tiles at $90 \mathrm{~W}$ in $20 \mathrm{ml}$ DI water for $1 \mathrm{~min}$ ute. $0.1 \mathrm{ml}$ of the bacteria suspension after sonication was enumerated as mentioned before. A zero hour reading taken immediately after initial bacteria inoculation on selected tiles was acquired to estimate the number of bacterial cells that can be recovered from the tile. The tiles were then exposed to visible light under various filters to assess the performance of the coatings at the limits of the absorption spectrum of the contaminant. The recovered microbes were then cultivated in TSA and enumerated using a colony counter. Effect of sonication on bacterial viability was also assessed to eliminate possible inactivation due to sonication

$$
\% \text { Bacterial Inactivation }=\frac{C_{0}-C_{t}}{C_{t}} * 100
$$

Where $C_{0}$ is the concentration of bacteria in CFU at time $t=0$ and $C_{t}$ is the concentration of the bacteria at an exposure of time' $t$ '. 


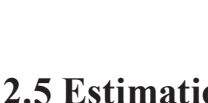

\section{Estimation of dye degradation}

Tiles coated with anatase were inoculated with $0.002 \mathrm{ml}$ of mordant orange at a concentration of $100 \mathrm{ppm}$. The tiles were then exposed to a light intensity of about $\sim 1.8 \mathrm{~W} / \mathrm{m}^{2}$ for $24,48,72$ and $96 \mathrm{~h}$ at $23{ }^{\circ} \mathrm{C}$ and $40 \% \mathrm{RH}$. Reflectance of coated and uncoated tiles were measured with a spectrometer. Absorbance was calculated as the negative $\log _{10}$ of reflectance expressed as a fraction. Dye degradation was calculated by comparing the reflectance measurements of coated tiles at 0 hour exposure to the groups exposed to light.

$$
\% \text { dye degradation }=\frac{A_{0}-A_{t}}{A_{t}} * 100
$$

Where $A_{0}$ is the absorption of dye at time $t=0$ and $A_{t}$ is the absorption of the dye at an exposure of time' $t$ '.

\subsection{Effect of optical cut-off filters on the inactivation experiments}

Optical filters with cut-off wavelengths were chosen in accordance with the absorption spectrum of the dye and the bacteria. Anatase tiles inoculated with bacteria or dye and were placed under 400, 495 and $550 \mathrm{~nm}$ filters. Bacterial and dye degradation was calculated for an exposure of 18 and 24 hours respectively at $23{ }^{\circ} \mathrm{C}$ and $40 \% \mathrm{RH}$. The results were then plotted comparing the photocatalytic performance under different cut off filters.

\section{Results and discussion}

\subsection{Absorbance spectrum of mordant orange, anatase and $\mathrm{S}$. aureus}

The absorbance spectrum of mordant orange, $S$. aureus and anatase on a ceramic tile substrate was acquired using the spectrometer. Fig. 1 illustrates an absorption cut off for anatase at $380 \mathrm{~nm}$. The absorption of staphylococcus aureus and mordant orange extends well into the visible spectrum with a cut off at around $550 \mathrm{~nm}$.

The spectrum indicates that any absorption in the visible region is accounted for by the dye or the bacterium on the surface of anatase.

\subsection{Photocatalytic inactivation of Staphylococcus aureus}

Photocatalytic inactivation of S. aureus was estimated on coatings of anatase and silica. Anatase coating, kept in the dark, was included as a negative control. Test coatings were exposed to a light intensity of about $2 \mathrm{~W} / \mathrm{m}^{2}, 32{ }^{\circ} \mathrm{C}$, $40 \% \mathrm{RH}$ and an exposure time of $18 \mathrm{~h}$. The spectral dis-

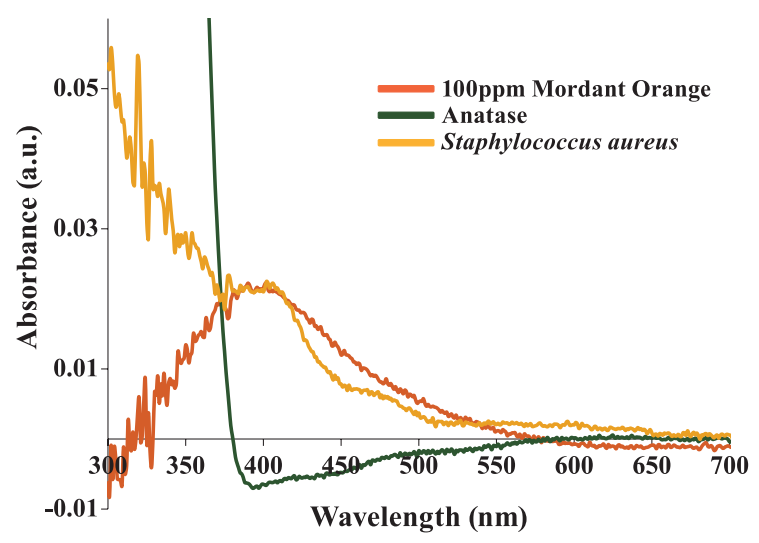

Fig. 1 Absorption spectrum of anatase (green), S. aureus (yellow) and Mordant Orange (Orange) across UV and visible spectrum obtained from reflectance measurements on ceramic tiles.

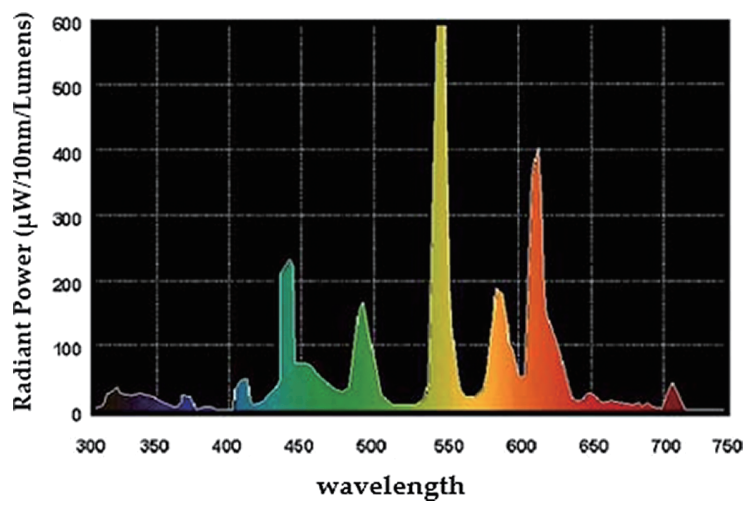

Fig. 2 Spectral distribution of the fluorescent lamp used for bacterial inactivation on tile surfaces. Image courtesy of General Electric Company (General Electric, 2016). Copyright: (2016) G.E.

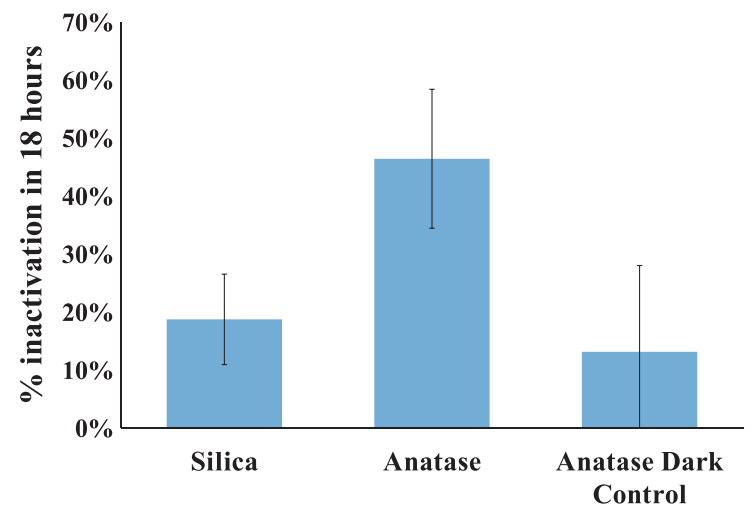

Fig. 3 Inactivation of $\mathrm{S}$. aureus on anatase, silica and in the dark over 18 hours at $23{ }^{\circ} \mathrm{C}$ and $40 \% \mathrm{RH}$.

tribution of the fluorescent lamp used for the experiment is shown in Fig. 2 (GeneralElectric). A $400 \mathrm{~nm}$ filter was used for all visible light photocatalysis to eliminate the small amount of UV present in the luminescent bulb spectra. Fig. 3 shows about $45 \%$ photocatalytic inactivation of $S$. aureus on unmodified anatase surfaces. The in- 
adeivatif lower and are attributed to desiccation of microbes over the period of $18 \mathrm{~h}$. The experiment indicates a synergy between the bacteria and the anatase surface introducing photocatalysis on anatase surfaces.

The lower inactivation on the silica and dark surfaces also eliminates the contribution of photolysis or oxidative degradation of dye under experimental conditions.

\subsection{Contribution of bacteria and dye (contaminant) to the process of visible light photocatalysis}

The contribution of contaminants to the process of visible light induced photocatalysis was studied by measuring the inactivation of $S$. aureus as the model bacterium and 5-(4-Nitrophenylazo) salicylic acid, (mordant orange) as a model dye. The kinetics of degradation for mordant orange on unmodified anatase surface were studied as a model for the bacteria. The dye was placed under long pass filters with exposure for 24, 48, 72 and 96 hours. The absorption spectrum of mordant orange exhibits a peak at $410 \mathrm{~nm}$ and gradually decreases with a cut off at $550 \mathrm{~nm}$ as shown in Fig. 1. Long pass filters cut off wavelengths at $400 \mathrm{~nm}, 495 \mathrm{~nm}$ and $550 \mathrm{~nm}$ filters were chosen to ensure non-interference of UV light (400 nm filter), reduced absorbance of dye $(495 \mathrm{~nm})$ and cut off of dye absorption (550 nm).

Dye degradation data was analyzed by comparing the reflectance data of dye at the start of the experiment to the tiles at different time points of the experiment. Results in Fig. 4 illustrate a reduction in photocatalytic activity of anatase surfaces with increasing wavelength cut off filters. A sharp decrease in the photocatalytic activity at the 495 filter is seen due to significantly decreased participation of the dye in the process. The photocatalytic degradation under a $550 \mathrm{~nm}$ is comparable to the dark control

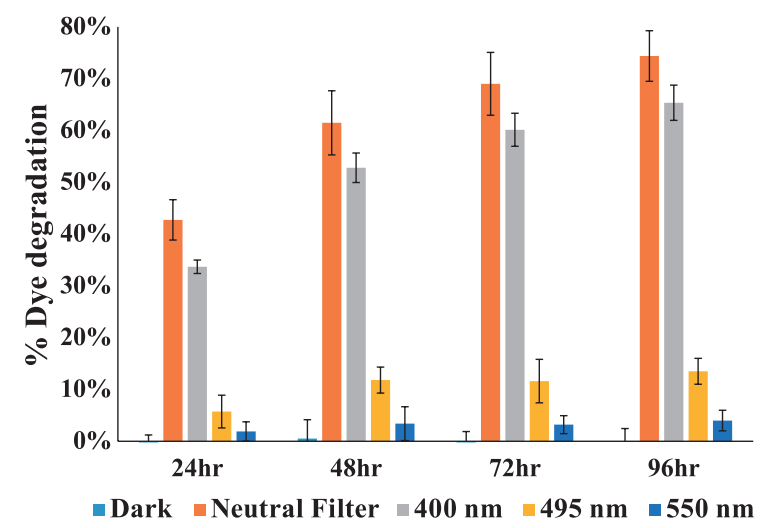

Fig. 4 Degradation of dye on anatase coatings over a period of $24 \mathrm{~h}, 48 \mathrm{~h}, 72 \mathrm{~h}$ and $96 \mathrm{~h}$ under various optical filters. The absorption of the dye clearly sensitizes the visible light photocatalysis of anatase. indicating no participation of dye in the process. The reduction in light intensities under various filters were recorded and show a maximum reduction of $5 \%$ at the $495 \mathrm{~nm}$ cut off and $16 \%$ at the $550 \mathrm{~nm}$ cutoff wavelengths. Subsequently, inactivation of $S$. aureus on anatase surfaces was carried out under similar spectral conditions to validate the mechanism of photocatalytic inactivation with biological contaminants. The results plotted in Fig. 5 show that $S$. aureus inactivation under visible light gradually decreased with increasing cut off limits up to $550 \mathrm{~nm}$ in accordance with dye degradation results. It is also noted from the absorption spectrum in Fig. 1 that the only component absorbing in the visible spectrum is the bacteria. Additionally, the trend of bacterial inactivation on anatase in visible light follows the trend in absorption intensity of bacteria. This indicates the antibacterial potential of unmodified anatase with visible light.

From the data obtained we hypothesize that the mechanism of microbial inactivation on unmodified anatase surfaces is similar to that of $\mathrm{TiO}_{2}$ dye sensitized solar cells (DSSC). In $\mathrm{TiO}_{2}$ based DSSC's irradiation with visible light leads to (i) excitation of the dye molecule, (ii) subsequent oxidation of dye molecule, and (iii) transfer of electrons from the dye molecule to the $\mathrm{TiO}_{2}$ conduction band. The electrons then diffuse through the circuit to the counter electrode. The electrolyte is reduced from $\mathrm{I}_{3}{ }^{-}$to $\mathrm{I}^{-}$ which diffuses and combines with the oxidized dye molecule to complete the circuit. Similarly in a contaminant activated photocatalyst, the dye or microbe acts as the electron source when irradiated with visible light. Fig. 6 is a schematic of the hypothesized mechanism of action on unmodified anatase surfaces when in contact with or-

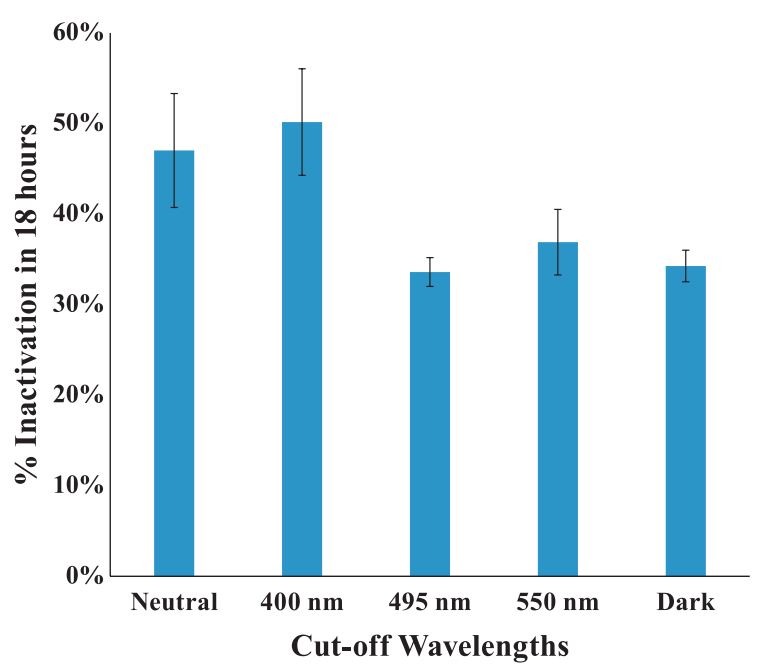

Fig. 5 Inactivation of S. aureus on anatase under different optical filters over 18 hours at $23{ }^{\circ} \mathrm{C}$ and $40 \% \mathrm{RH}$. The inactivation of bacteria decreases with the increase in cut off wavelengths of the long pass filters from 400 to $550 \mathrm{~nm}$. 


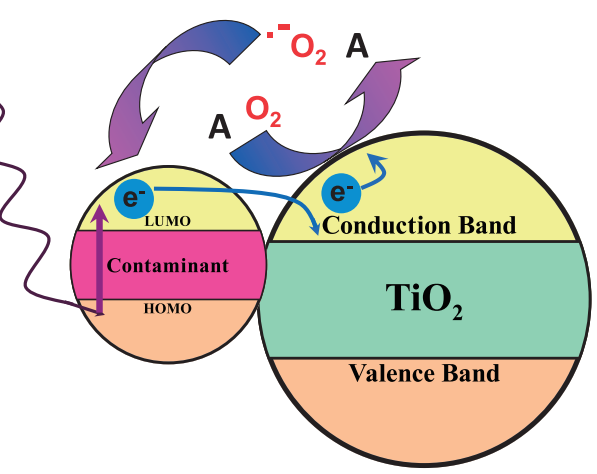

Fig. 6 Schematic for possible mechanism of inactivation on unmodified $\mathrm{TiO}_{2}$ surfaces (Krishna et al., 2016).

ganic contaminants such as microbes(Krishna et al., 2015). Electrons inserted in the conduction band of $\mathrm{TiO}_{2}$ catalyze the small molecules like oxygen and water into super oxide free radicals. These reactive radicals in turn oxidize most organic molecules to $\mathrm{CO}_{2}$ and water. The generation of free radicals in the photocatalyst with availability of microbial contaminants leads to their continued inactivation.

\section{Conclusions}

The present study illustrates the potential of unmodified nano-crystalline anatase as a photocatalytic disinfectant in visible light when in contact with microbes. While the kinetics of inactivation of bacteria on such unmodified surfaces is not as fast as in the case of conventional disinfectants, photocatalytic coatings can last much longer and have a very high cumulative contribution towards surface disinfection. Masking by inactivated microbes often hampers the performance of active coatings by forming a barrier between the active species and the microbes. However, the complete degradation of microbes (organic contaminants) on anatase surfaces ensures no 'masking effect' unlike on other potent antimicrobial surfaces like copper and silver. Thus, anatase surfaces in comparison can function for long inactivation periods. We would also like to further indicate that these coatings can be applied to surfaces and complemented with additional disinfection treatments to have increased efficacies of surface disinfection. A combination of disinfection approaches can be used for surfaces that do not receive large amounts of UV light and kill pathogens that are transferred through touch mediation.

\section{Acknowledgements}

The authors would like to acknowledge the National Science Foundation Grant No. 1127830 for their financial support and the industry members of the Centre for Particulate and Surfactant Systems (CPaSS) for their help and contribution to this project. Experimental work was carried out the University of Florida Water Reclamation and Reuse laboratory and at the Particle Science and Technology Laboratory. We would like to thank Dr. Wei Bai for the SEM micrographs.

\section{Nomenclatures}

$\begin{array}{ll}\text { CFU } / \mathrm{ml} & \text { Colony forming units per } \mathrm{ml} \\ A_{0} & \text { Absorbance at time } 0 \\ A_{t} & \text { Absorbance at time } t \\ C_{0} & \text { Concentration at time } 0(\mathrm{CFU} / \mathrm{ml}) \\ C_{t} & \text { Concentration at time } t(\mathrm{CFU} / \mathrm{ml}) \\ \mathrm{RCF} & \text { Relative Centrifugal Force } \\ \mathrm{RH} & \text { Relative Humidity }(\%) \\ \mathrm{RPM} & \text { Rotations per minute } \\ \mathrm{W} / \mathrm{m}^{2} & \text { Watt/ Square meter }\end{array}$

\section{References}

Asahi R., Morikawa T., Ohwaki T., Aoki K., Taga Y., Visiblelight photocatalysis in nitrogen-doped titanium oxides, Science, 293 (2001) 269-271.

Bach U., Lupo D., Comte P., Moser J.E., Weissortel F., Salbeck J., Spreitzer H., Gratzel M., Solid-state dye-sensitized mesoporous $\mathrm{TiO}_{2}$ solar cells with high photon-to-electron conversion efficiencies, Nature, 395 (1998) 583-585.

Bai W., Krishna V., Wang J., Moudgil B., Koopman B., Enhancement of nano titanium dioxide photocatalysis in transparent coatings by polyhydroxy fullerene, Appl. Catal. B-Environ., 125 (2012) 128-135.

Chen F.N., Yang X.D., Wu Q., Antifungal capability of $\mathrm{TiO}_{2}$ coated film on moist wood, Build. Environ., 44 (2009) 1088-1093.

Erkan A., Bakir U., Karakas G., Photocatalytic microbial inactivation over Pd doped $\mathrm{SnO}_{2}$ and $\mathrm{TiO}_{2}$ thin films, J. Photoch. Photobio. A, 184 (2006) 313-321.

Fujishima A., Zhang X.T., Tryk D.A., $\mathrm{TiO}_{2}$ photocatalysis and related surface phenomena, Surf. Sci. Rep., 63 (2008) 515582.

GeneralElectric, Spectral Power Distribution Curves: TriPhosphor Fluorescent Colors, SPX41. Commercial Products and Solutions. http://www.gelighting.com/LightingWeb/na/ resources/tools/lamp-and-ballast/pop_curves.jsp?15, accessed 06.06.2016.

Henderson M.A., A surface science perspective on $\mathrm{TiO}_{2}$ photocatalysis, Surf. Sci. Rep., 66 (2011) 185-297.

Ishibashi K., Fujishima A., Watanabe T., Hashimoto K., Quantum yields of active oxidative species formed on $\mathrm{TiO}_{2}$ photocatalyst, J. Photoch. Photobio. A, 134 (2000) 139-142.

Klein E., Smith D.L., Laxminarayan R., Hospitalizations and deaths caused by methicillin-resistant Staphylococcus aureus, United States, 1999-2005, Emerg. Infect. Dis., 13 (2007) 1840-1846.

Konaka R., Kasahara E., Dunlap W.C., Yamamoto Y., Chien 
K. I. Inoue M., Irradiation of titanium dioxide generates Wboth singlet oxygen and superoxide anion, Free Radical Bio. Med., 27 (1999) 294-300.

Krasovec U.O., Berginc M., Hocevar M., Topic M., Unique $\mathrm{TiO}_{2}$ paste for high efficiency dye-sensitized solar cells, Sol. Energ. Mat. Sol. C, 93 (2009) 379-381.

Krishna V., Bai W., Han Z., Thakur A., Koopman B., Moudgil B., Contaminant-Activated Visible Light Photocatalysis for Reducing Healthcare-Acquired Infections, In review, Nature (2016).

Lilja M., Forsgren J., Welch K., Astrand M., Engqvist H., Stromme M., Photocatalytic and antimicrobial properties of surgical implant coatings of titanium dioxide deposited though cathodic arc evaporation, Biotechnol. Lett., 34 (2012) 2299-2305.

Linsebigler A.L., Lu G.Q., Yates J.T., Photocatalysis on $\mathrm{TiO}_{2}$ Surfaces-Principles, Mechanisms, and Selected Results, Chem. Rev., 95 (1995) 735-758.

Lobdell K.W., Stamou S., Sanchez J.A., Hospital-Acquired Infections, Surg. Clin. N. Am., 92 (2012) 65-77.

Mitoraj D., Janczyk A., Strus M., Kisch H., Stochel G., Heczko P.B., Macyk W., Visible light inactivation of bacteria and fungi by modified titanium dioxide, Photoch. Photobio. Sci., 6 (2007) 642-648.

Oregan B., Gratzel M., A Low-Cost, High-Efficiency Solar-Cell Based on Dye-Sensitized Colloidal $\mathrm{TiO}_{2}$ Films, Nature, 353 (1991) 737-740.

Otter J.A., Yezli S., Perl T.M., Barbut F., French G.L., The role of 'no-touch' automated room disinfection systems in infection prevention and control, J. Hosp. Infect., 83 (2013) $1-13$.

Scott II R.D., The Direct medical costs of healthcare-associated infections in U.S. hospitals and the benefits of prevention, Centers for Disease Control and Prevention (2009). http:// stacks.cdc.gov/view/cdc/11550/, accessed 06.06.2016.

Shen M., Wu Z.Y., Huang H., Du Y.K., Zou Z.G., Yang P., Carbon-doped anatase $\mathrm{TiO}_{2}$ obtained from TiC for photocatalysis under visible light irradiation, Mater. Lett., 60 (2006) 693-697.

Sunada K., Watanabe T., Hashimoto K., Studies on photokilling of bacteria on $\mathrm{TiO}_{2}$ thin film, J. Photoch. Photobio. A, 156 (2003) 227-233.

Tucci P., Porta G., Agostini M., Dinsdale D., Iavicoli I., Cain K., Finazzi-Agro A., Melino G., Willis A., Metabolic effects of $\mathrm{TiO}_{2}$ nanoparticles, a common component of sunscreens and cosmetics, on human keratinocytes, Cell Death Dis., 4 (2013).

Weber D.J., Anderson D., Rutala W.A., The role of the surface environment in healthcare-associated infections, Curr. Opin. Infect. Dis., 26 (2013) 338-344.

Wei X.J., Yang Z.D., Tay S.L., Gao W., Photocatalytic $\mathrm{TiO}_{2}$ nanoparticles enhanced polymer antimicrobial coating, Appl. Surf. Sci., 290 (2014) 274-279.

Yu J.C., Xie Y.D., Tang H.Y., Zhang L.Z., Chan H.C., Zhao J.C., Visible light-assisted bactericidal effect of metalphthalocyaninesensitized titanium dioxide films, J. Photoch. Photobio. A, 156 (2003) 235-241.

\section{Author's short biography}

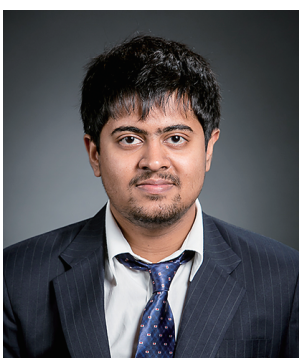

Vignesh Nandakumar

Vignesh Nandakumar is a third year graduate student in the Materials Science and Engineering at the University of Florida. His Scientific activities and research interests focus on understanding cell materials interactions, and designing systems that can manipulate surface properties of cells to remove, kill or enhance adhesion onto surfaces. Vignesh received his Bachelors from SASTRA University, Tanjore, India and his M.S. in Material Science and Engineering from the University of Florida, Gainesville, Florida.

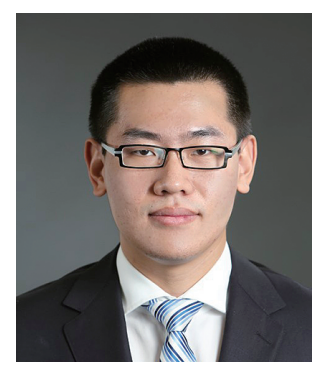

\section{Zhao Han}

Zhao Han is an Analytical Chemist/Material Scientist of Western Digital (WD) located in San Jose, California. Zhao is a graduate of University of Florida, where he received a M.S. in Materials Science and Engineering (MSE) 2013. He also received his B.S. in MSE from Beijing University of Technology 2011 with expertise in Metal Processing. During his graduate study, Zhao researched on visible light activated $\mathrm{TiO}_{2}$ antimicrobial coating and developed the mechanism of contaminant activation by using optical cut-off filters and organic dye. After graduation, Zhao started his professional career at WD and focuses on hard disk drive development. 


\section{Author's short biography}
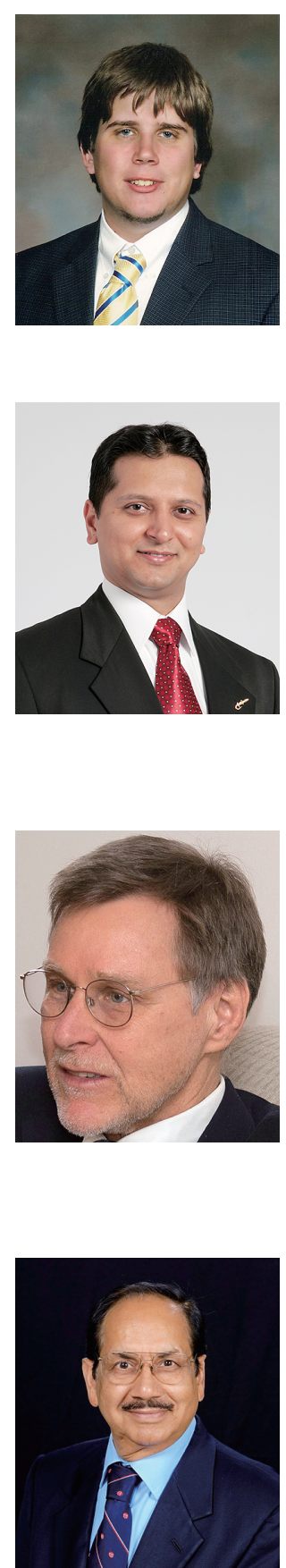

\section{Zachary Fritz}

Zachary Fritz is a research scientist at a food-testing biotech company and will be pursuing graduate studies in Biomedical Engineering at Rutgers University (New Brunswick, New Jersey). His research interests include immunological engineering and tissue engineering. He received his B.S.'s in Chemical Engineering and Microbiology from the University of Florida (Gainesville, Florida).

\section{Vijay Krishna}

Vijay Krishna, Ph.D., was a Postdoctoral Fellow with the Particles Engineering Research Center at the University of Florida. Dr. Krishna has been awarded 6 patents and is primary inventor on 12 current patent applications. He has authored 16 journal publications and presented at more than 30 technical conferences. Dr. Krishna earned a Ph.D. in Materials Science and Engineering at the University of Florida. He is currently a Project Scientist at the Lerner Research Institute, Cleveland Clinic. His research interests include carbon nanostructures, bio-nano interactions, surface and colloid science.

\section{Ben Koopman}

Ben L. Koopman, Ph.D., is a Professor and Undergraduate Coordinator in the Department of Environmental Engineering Sciences at the University of Florida. He earned a B.S. in Civil Engineering from Oregon State University before pursuing a Ph.D. in the same field at the University of California, Berkeley. His research interests include removal of microorganisms by surface-modified filter media and bio films, photocatalytic antimicrobial coatings, water treatment, one-dimensional modelling of activated sludge secondary settling tanks, and biological nutrient removal.

\section{Brij M. Moudgil}

Dr. Brij M. Moudgil is a Distinguished Professor of Materials Science and Engineering at the University of Florida. His current research interests are in particulate materials based systems for enhanced performance in bioimaging, nanomedicine, photocatalytic degradation of hazardous microbes, polymer and surfactant adsorption, dispersion and aggregation of fine particles and nanotoxicity. Dr. Moudgil received his B.E from the Indian Institute of Science, Bangalore, India and his M.S and Eng.Sc.D degrees from Columbia University, New York. He has published more than 400 technical papers and has been awarded 14 patents. He is a member of the U.S National Academy of Engineering. 